\title{
Lessons from coordinating a knowledge- exchange network for connecting research, policy and practice
}

\author{
Tanya Wilkins* - Australian National University, Australia \\ Ian Cooper - Eclipse Research, Cambridge, UK
}

\begin{abstract}
This article explores ten lessons learnt by the UK knowledge-exchange network, the Adaptation and Resilience in the Context of Change (ARCC). The lessons signpost the importance of 'soft skills' required for the development of effective knowledge exchange and indicate the need for a broad definition of knowledge exchange that allows for a flexible approach to meet the diverse and changing needs of research, policy and practice. Such flexibility will also encompass collaborative research conducted with business, consultancy, training, start-ups and commercialization components. The ten lessons here will be valuable to: networks and individual academics undertaking knowledge exchange to achieve research excellence and impact with non-academic audiences; those undertaking public engagement and stakeholder engagement with research; and government policymakers and research managers tasked with shaping and implementing the UK's proposed Knowledge Exchange Framework (KEF).
\end{abstract}

Keywords: knowledge exchange, impact, non-academic audiences, communication, outreach, engagement, soft skills, intangibles

\section{Key learning points}

- The Adaptation and Resilience in the Context of Change (ARCC) network championed the premise that knowledge exchange between academic researchers and policy and practice communities is crucial to delivering research benefits across the UK economy and society.

- A careful balance is required between the various types of engagement provided to allow members of a knowledge-exchange network to choose what to access and when - depending on their current level of interest and capacity to absorb what is on offer.

- Done well, the work of a knowledge-exchange coordination team is often invisible, because of its emphasis on the deployment of soft skills, and on the exchange of social capital among and between diversely situated network members. 


\section{About Adaptation and Resilience in the Context of Change (ARCC)}

The UK Climate Impacts Programme (UKCIP), located at the Environmental Change Institute at the University of Oxford, was continuously funded by the UK's Engineering and Physical Sciences Research Council (EPSRC) to support the Adaptation and Resilience in the Context of Change (ARCC) network from 2009 until 2018. The network had three key objectives:

- building community cohesion to develop in-depth understanding and synergies across the network

- provision and integration of knowledge to help ensure policy and practice have the best available evidence

- enhanced accessibility and uptake of research outputs to meet the needs of a diverse stakeholder community in a timely manner.

The role of the network was to engage with researchers in the built environment and infrastructure sectors to provide them with a comprehensive and coordinated focal point for their knowledge-exchange activities. For the best part of a decade, the network sought to promote opportunities for academic researchers to engage with significant policy and practice communities that need access to evidence produced by researchers to assist their decision-making processes.

The network's coordination team built up a wealth of experience and expertise in managing a knowledge-exchange network, and facilitating stakeholder engagement processes and knowledge-exchange activities. When the activities of the ARCC network coordination team was reviewed by an EPSRC Review Panel in 2014, its work on stakeholder engagement and knowledge exchange was judged to be 'exemplary'. This commentary records the subsequent journey of self-examination that the team embarked upon in order to capture how they had achieved this. Their examination focused not only on the team's own knowledge and experience, but also on the lessons they had learnt for delivering knowledge exchange by providing separate but complementary advice for knowledge-exchange professionals, academics, publicengagement practitioners and communication managers within higher education institutions.

In the exchange of knowledge, networks have been identified as potentially effective mechanisms for dissemination and application because their 'principal purpose is to connect people who might not otherwise have an opportunity to interact, enable dialog, stimulate learning, and capture and diffuse knowledge' (Gagnon, 2011: 27). These mechanisms include communities of practice, knowledge networks and soft networks. The ARCC network was focused specifically on increasing the impact of research investments in the built environment and infrastructure sectors. However, many of the approaches adopted by the coordination team are transferable, and are of value across a broad range of sectors, for example:

- making connections at all levels and across boundaries is paramount to enhancing value

- underlying the successful operation of the network is the effective exchange of social capital, both through formal activities and by taking advantage of unexpected, less formal opportunities

- helping all members of the network enhance their involvement by being clear, not only in what they are looking for, but in what they have to offer, what is of value, and how they wish this to be judged 
- offering opportunities to develop engagement and knowledge-exchange skills is valued at all stages of career development - such skills and learning are highly transferable

- identifying and meeting the very diverse needs of the network members requires a flexible approach and an array of technical and interpersonal skills within the coordination team

- moderating the balance between various forms of engagement is a key task of the coordination team, allowing members to choose what to access depending on their own level of interest and absorptive capacity

- done well, the exercise of such tasks, and the skills needed to support them, by the coordination team was often invisible but, to work effectively, required a substantial level of investment to provide strong and energized support to members.

The self-reflective review of the ARCC network by members of the coordination team revealed how their own needs had changed over time. Their initial needs were based on discipline-specific and technical knowledge in order to inform research project coordination. As the research projects developed, team members required broader knowledge-exchange and communication skills to assist researchers and their industrial partners in synthesizing and disseminating research outputs. Then they had to focus explicitly on showcasing the research agenda that underpinned the majority of ARCC activities over the final 18 months of the ARCC network.

\section{The UK's evolving approach to knowledge exchange}

UK research councils have actively encouraged activities - such as stakeholder engagement, knowledge exchange and pathways to impact - that enhance the impact of the research they fund. Like other funding agencies, they are concerned to show that their investments deliver 'value for money'. For instance, a study by the Higher Education Funding Council for England (HEFCE, 2015) suggested that every pound

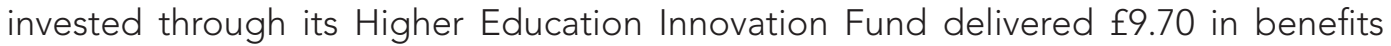
for the economy and society. The funding of the ARCC network by the UK's EPSRC reflected a growing investment in, and emphasis on, the importance of knowledge exchange by the UK's research councils. In the UK, academics, knowledge-exchange professionals, policymakers and practitioners are now expected to actively interact with potential users of their research.

Investment in knowledge exchange and dissemination by UK research councils pre-dates the UK's current Research Excellence Framework (REF, 2019) and its forerunner, the Research Assessment Exercise (RAE, 2008). Indeed, the founding royal charter (1965) of what is now the EPSRC already outlined the dual role of grant holders to carry out their research and to disseminate their findings (Cooper, 1993).

The REF has continued to encourage knowledge-exchange activities in UK higher education (HE) institutions. It has incentivized the communication and commercialization of research through the requirement for comprehensive peerreviewed impact case studies (REF, 2019). However, what was seen as underperformance in commercialization of research in the UK led the government to propose a new Knowledge Exchange Framework (KEF) to help the HE system 'as a whole to find a new gear' (Johnson, 2017: n.p.).

What the 'new gear' ushered in by the KEF might turn out to be cannot be easily answered until there is a shared understanding of the definition being implemented for knowledge exchange itself. Work on the KEF is being led by United Kingdom Research 
and Innovation (UKRI), a newly founded organization in operation since 1 April 2018. It brings together the UK's seven existing research councils, as well as Innovate UK and the research and knowledge-exchange functions of HEFCE, in one unified body to direct research funding (BIS, 2016). It aims (UKRI, n.d.(b)) to:

... support and encourage universities and other research institutions to make KE [knowledge exchange] and commercialisation an equal priority alongside their teaching and research missions ... [in order] to enable all parts of the UK to realise their potential for innovation-led growth.

UKRI has committed itself to use its new KEF to provide more information for the public and businesses on the performance of universities in knowledge exchange - how they share knowledge, expertise and other assets for the benefit of the economy and society. How this is likely to unfold is signalled by the consultation on the forthcoming KEF launched by Research England (2019) with proposed metrics based around seven 'perspectives': research partnerships; working with business; working with the public and the third sector; skills, enterprise and entrepreneurship; local growth and regeneration; intellectual property and commercialization; and public and community engagement.

\section{The meaning of 'knowledge exchange'}

The ARCC network explored the scope and purpose of knowledge exchange as part of its own development and implementation activities. Unsurprisingly, it uncovered many definitions of knowledge exchange, supported, for instance, by a Google search for 'knowledge exchange definition' that returned 345 million results (in January 2019). Both grey and academic literature suggest a range of interpretations of 'knowledge exchange'. These differentiate between the 'provision of information', 'knowledge transfer' and 'dissemination' at one end of the spectrum, and 'knowledge exchange' - which refers to the two-way provision of information between researchers and stakeholders - at the other. In parallel, there is the more commercially driven vocabulary of 'technology transfer', and 'spin out' companies. In the ongoing discussion in the UK about how to frame the KEF, it will be important not to use these terms as if they are synonyms, for their meanings - and the activities required to support them - are highly nuanced.

The health sector emerges as the most active in pursuing the meaning and application of knowledge exchange. A decade ago, Tetroe et al.'s study (2008) of the health sector identified 33 applied research funding agencies in nine countries active in knowledge-exchange funding, with 29 terms being used to refer to some aspect of the concept of turning knowledge into action. A definition (Fazey et al., 2013: 19) that the ARCC coordination team applied to its own work with the ARCC network is knowledge exchange as a process that 'generates, shares and/or uses knowledge through various methods appropriate to the context, purpose, and participants involved'. The coordination team also particularly appreciated the identification of knowledge exchange employed by the National Endowment for Science, Technology and the Arts (NESTA) - an independent innovation foundation based in the UK - as 'an extra nudge, or a helping-hand' in order to get research used by decision makers (Breckon and Dodson, 2016: 4). NESTA's study of the literature on knowledge exchange identified over 150 possible interventions employed to help get research used, including professional recognition, social marketing, championing evidence with decision makers, finding out what questions need answering by decision makers, and 
ensuring that campaigns are targeted at what the relevant people care about. Clearly there are a plethora of ways and means of promoting knowledge exchange, and this diversity reflects hard-won experience that achieving effective knowledge exchange is, in practice, no small task, with no 'one-size-fits-all' solution.

\section{Knowledge exchange in the ARCC network}

As knowledge-exchange specialists, the coordination team for ARCC network advocated and worked to raise the profile of knowledge exchange during its eight years of operation, for example through its bespoke training for researchers on how to manage stakeholder relationships and on how to respond to the 'pathways to impact' agenda. One of the ways in which knowledge-exchange coordinators can add value to the results of academic research is by using their trusted and impartial role to bring together the research, policy and practice communities that need to collaborate in order to make the uptake of academic research more effective. Here, the ARCC coordination team acted as a 'cheerleader' for research by supporting the collaboration between its members from research, policy and practice in order to help create robust built environment and infrastructure sectors.

The ARCC network operated as an explicit knowledge 'exchange'. It had its own distinct agenda and mode of operation by providing a safe space for mutually supportive knowledge-exchange activities that could be jointly occupied by researchers, intermediaries and the users of their research results, rather than just concentrating on promoting commercialization or spin-out. This differentiated it from the UK's Knowledge Transfer Network (KTN, 2019) - which 'helps businesses get the best out of creativity, ideas and the latest discoveries, to strengthen the UK economy and improve people's lives', and from Innovate UK - a non-departmental public body operating at arm's length from the government as part of the UKRI - 'with [its] strong business focus, [to] drive growth by working with companies to de-risk, enable and support innovation' (UKRI, n.d.(a)). Nor did the space created by the ARCC network seek to duplicate individual higher education institutions' own commercialization centres, such as Oxford University Innovation Ltd, a wholly owned subsidiary of the University of Oxford that manages its technology transfer and consulting activities (Oxford University Innovation, n.d.).

While the commercialization of research may result in whole new products, technologies or industries, there is a quite different approach required to support the development of useable science for decision making (Dilling and Lemos, 2011). Originally, ARCC positioned itself as a boundary organization that acted primarily across a specific EPSRC-funded (climate change) research portfolio. Initially, it sought to bring together related research investment to exploit synergies and complementarities and, subsequently, to collate emerging research results as a synthesized and cohesive evidence base that could be used more readily for policy and practical decision making. While maintaining its role at the boundary of research/policy/practice, ARCC evolved into a coordination hub showcasing multiple research projects across several research themes and programmes, taking research directly to the stakeholders, for example at leading trade shows such as the UK's Construction Week or Ecobuild exhibition and conference. The coordination team also played a role, for instance in understanding the research needs of infrastructure operators in the UK and in showcasing research to support government departments to further their understanding of the research capacity available across UK universities that could support their policy formulation and implementation. These knowledge-exchange activities, often perceived as 'softer' 
and more expansive, grew alongside the original remit given to the network, and did so with the blessing of the EPSRC that funded it.

During the life of the ARCC network, further challenges were raised by both the UK's Research Excellence Framework (REF), which put the responsibility for demonstrating the impact of research projects on to the higher education institutions that housed them (REF, 2019), and also through the introduction of the Pathways to Impact initiative (of the Research Councils UK, now UKRI), which placed responsibility for demonstrating impact from research projects, in the first instance, on grant holders (RCUK, 2010). In response, the ARCC network set out to provide additional collective expertise, offered through its collaborative non-competitive space, to share skills, perspectives and experience for delivering knowledge exchange. This sharing was critical for enabling publicly funded research projects to maximize and accelerate their impact - both individually and through pooled research results. The work of the ARCC network has been acknowledged as both 'welcomed' and 'trusted' by its academic and non-academic stakeholders (UKCIP and Cooper, 2016). And the importance of the continuation of such managed knowledge-exchange activities has been recognized: 'it is crucial that longer-term, yet flexible, mechanisms to support the exchange of knowledge and evidence are established to enhance the value built up by investments in research' (ibid.: 5).

\section{Assembling ARCC lessons for successful knowledge exchange}

When the EPSRC (2014) Review Panel judged the work of the ARCC network coordination team as being 'exemplary', it issued a challenge to the team to capture exactly how it had operated so effectively to support stakeholder engagement and knowledge exchange. This challenge was issued in order to identify lessons that could help improve the performance of other knowledge-exchange networks and the management of other large-scale research programmes. In practice, the team did not find it easy to respond to this challenge. Up until that point, the team had been operating on the basis of its members' tacit knowledge and their experience-based learning - which they had not previously attempted to share or capture.

The approach adopted for trying to capture lessons learnt was (seemingly) straightforward. Members of the coordination team were simply encouraged - through workshops, individual interviews and at-a-distance dialogues - to say out loud or to write down plainly what they knew about how the team had operated. This approach was based on one of the assumptions that underpins 'action learning' (Revans, 1982; Trehan and Pedler, 2011) - that, between them, team members had sufficient experience and expertise to identify what made their coordination practices and activities 'exemplary'. By actively listening (Robertson, 2005) to each other, and through acting as reflective practitioners (Schön, 1983), they were encouraged to turn their implicit appreciation into a more explicitly expressed understanding of the strategies, tactics and practices they had been employing. The members of the coordination team captured messages that were relevant to: (1) maintaining and operating a network; (2) network members themselves; and (3) funding agencies.

In parallel, selected academic and non-academic members of the knowledgeexchange network were asked what they valued about it and what they wanted it to deliver on their behalf. These network members were not chosen to be representative of the network's membership (over a thousand individuals); instead, they were selected to illustrate the widely different types of stakeholder groups that the coordination 
team served. Their responses (UKCIP and Cooper, 2016) illustrate the highly complex and differentiated sets of demands imposed on the coordination team by the very wide range of stakeholders that they were attempting to support - research funders, (government) policymakers, sector-based forums, professional institutions, established academic researchers, early career researchers and private sector design consultants. The responses from these types of stakeholder made up the first part of the report produced on the lessons learnt (ibid.: 9-20). The second part outlined the strategies and tactics that coordination team members developed or adopted for dealing with this complexity (ibid.: 21-34).

The results of these interviews (UKCIP and Cooper, 2016) illustrated the highly disparate array of needs, wants, expectations and aspirations that the coordination team tried to serve. Each type of stakeholder employed a slightly different set of criteria for judging how successful they thought the network and its coordination team had been. These differences illustrate how sensitive coordination team members needed to be when attempting to provide support and services to each type of stakeholder group - especially when dealing simultaneously with mixtures of them, either at the same event or through the same report or publication.

As its report revealed, the team achieved its success by observing their wide range of stakeholder groups carefully, listening to them attentively, and trying to empathize - seeking to put themselves in the positions of those they were providing with services or who needed to understand the practice-orientated outputs they were helping to develop. Doing this effectively required team members to deploy not just technical capabilities, but also an extensive set of interpersonal competencies - soft skills that are not normally seen as being at the forefront of requirements for delivering engineering-related research. Ironically, when practised well, these skills appeared to be largely invisible to network members.

Through their reflection on how the ARCC knowledge-exchange network had operated, the members of the coordination team managed to foreground the often hidden and taken-for-granted characteristics of knowledge exchange. This 'softer side' of knowledge exchange involves, for example, the translation, communication and showcasing of research in order to support the non-academic impact of research. Capturing the soft skills exercised by members of the coordination team - just like capturing the soft evidence of how and why its members valued the ARCC network required a social constructivist approach, where knowledge is seen as being generated between people interacting in specific social situations (Von Glaserfeld, 1989). This involved the collection of detailed verbatim statements from stakeholders and the use of grounded theory techniques (Glaser, 1993) to analyse the patterns detected in them. Unsurprisingly, the results generated cannot be reduced either to a simple 'one case fits all' or to easy one-liners. Instead, both network members' aspirations, and the skills that coordination team members deployed to meet them, were subtly complicated. Developing the skill set required to meet these aspirations required continuous major public investment, lasting over almost a decade. As both members of the ARCC network and of the coordination team indicated when interviewed (UKCIP and Cooper, 2016: 4), the fruits of such investments should not be abandoned because of the finite nature of research council funding initiatives. Instead long-term mechanisms for supporting knowledge exchange are required, if the value built up by such investment is not to be lost.

The ten key lessons listed below have been distilled from the ARCC coordination team's investigation into how they had sought to support the knowledgeexchange activities of the ARCC network over the best part of a decade (UKCIP and 
Cooper, 2016: 35-8). The insights that team members constructed from their reflective activities were captured in narratives using the team member's own words. These told their 'stories' of how the coordination team worked, and signalled what they saw as the significant lessons they had learnt over the lifetime of the ARCC knowledge-exchange network. It needs to be signalled as being particularly challenging for those mandated to support knowledge exchange that, when practised well, these skills - and the team members who practise them - often appear to be largely invisible to network members who are being supported by their proficient deployment.

\section{Listing the lessons learnt}

The following list is a synthesis of views contributed by current and former members of the ARCC coordination team, and by key stakeholders interviewed (see UKCIP and Cooper, 2016: 35-8).

\section{Lesson 1: Knowledge exchange cannot be treated as a trivial task}

The ARCC network learnt that translating the results from multiple research projects into digestible formats for non-academic audiences is not a trivial task. It calls for a high level of expertise that, in turn, requires dedicated investment of time and resources. There are specific strategies and tactics that members of the coordination team developed or adopted to deal with the complex set of demands imposed on them by the disparate needs, aspirations and motivations of the stakeholder groups that made up the ARCC network. Generated through interviews, the team listed key skills and/or capabilities that they felt were crucial to fulfilling their specific roles within the team. Box 1 lists all the skills that team members identified as necessary to support the effective management of knowledge-exchange activities.

Box 1: Skills identified that are required for effective knowledge exchange

Strategic leadership

Sector champion

Establishing credibility

Expert domain knowledge

Driving engagement

Inspiring confidence

Lateral thinking

Looking for implications

Finding knowledge gaps

Understanding wider context

Going beyond quantitative information

Establishing effective working relations

Exploiting expertise in related areas

Applying lessons between domains

Understanding policy drivers

Understanding of current policy

Technical understanding

Grasp of academic and funding requirements

Understanding industry practices

Awareness of industry's role in maximizing impact 


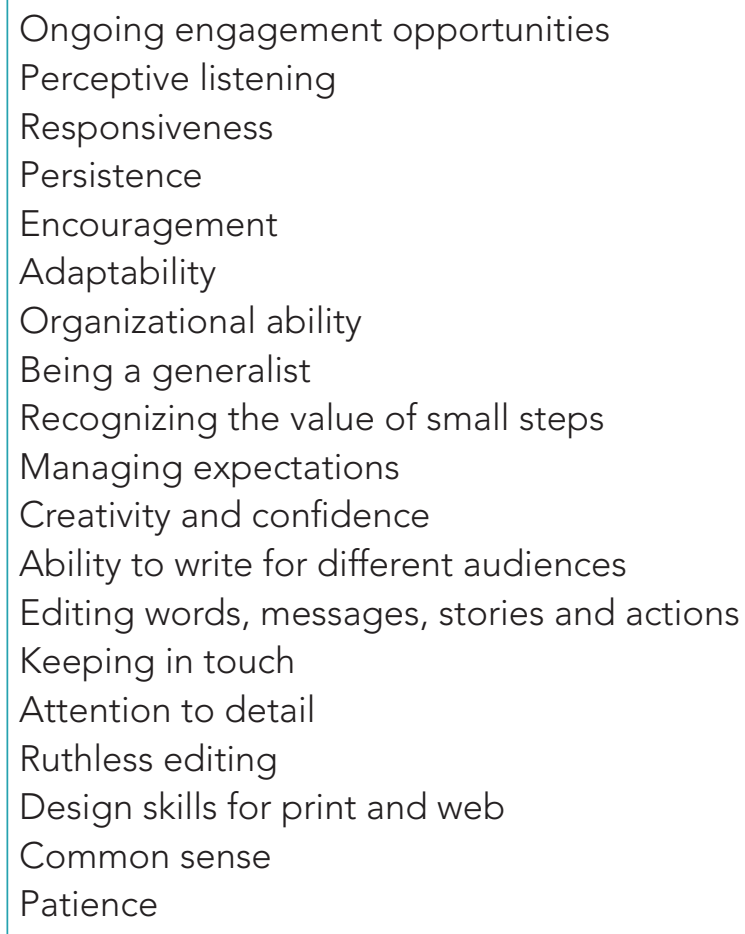

\section{Lesson 2: Building trust, credibility and openness}

Building and managing personal relationships with researchers and other stakeholders is crucially important, as is team members investing time and effort in understanding where the priorities of each type of stakeholder lie at any given point during their engagement. Resources need to be made available to support the time and energy that need to be invested, first in gaining and then in maintaining such trust. As with other 'soft' skills', it is not simple or easy to identify performance indicators that can be used to illustrate the value of such investment - yet it remains critical to the successful operation of a knowledge-exchange network.

\section{Lesson 3: Demonstrating value}

The ARCC network knew that a key and early priority was to demonstrate the value of the network to its stakeholder groups, including researchers, policymakers and practitioners, despite the different perspectives and values that each group applied when making its judgements. In its trusted and safe boundary role, the ARCC network was able to work with both researchers and with the policy and practice communities to enable two-way knowledge exchange. It is this two-way knowledge exchange, rather than a single, one-way, transfer, that is critical. More significant still can be the coconstruction of knowledge between these parties, which cannot be attempted without a neutral space for them to occupy and within which to build trust between them (and in the members of the coordination team).

\section{Lesson 4: Managing relationships}

To exploit their own technical expertise more effectively, what research teams required from a coordination team was help in how to engage and manage their appropriately targeted stakeholders. Much of a team's work involves improving the management of these relationships between researchers themselves, and between them and their non-academic partners. Such relationship management is needed so that they can 
work together effectively in order to realize both individual and collective benefits. Building this capacity in the researchers requires more than just exposing them to their non-academic partners; it also requires them to develop specific skills, such as being able to provide progress reports on the emerging results from their work in forms that are intelligible and meaningful to non-academics. The ARCC network was able to support such developments through their bespoke training programmes and through signposting which were the relevant stakeholder events for each researcher to attend.

\section{Lesson 5: Communicating effectively}

Each member of a coordination team needs to be an active and attentive listener. Listening attentively to what stakeholders say (and what they do not say) is a prerequisite for communicating with them effectively. This requires more than just an ability to stand up in meetings and talk about what a network can deliver for stakeholders. It also requires attention to stakeholders' non-verbal signals. And active listening and talking effectively to stakeholder groups then needs to be backed up by writing in clear, plain English. These skills have to be acknowledged as a necessary part of each team member's job, whatever their personal specialism. Each team member has to understand the importance of practising these communication skills, and of exploring the various opportunities that arise for putting them into practice. This necessarily includes focusing on who the audience is - and what their specific needs are - for each particular engagement activity/opportunity.

\section{Lesson 6: Making translations}

When working with researchers, it is important to be able to translate their research results into something that is deemed to be really useful for the policy and practice communities. This might include producing open-access summary papers, blogs or contributions to government consultations. Effective translation of the questions and concerns raised by practitioners, industrialists and members of the policy community in a form that researchers can act on is equally important. Such informed and sustained engagement is critical to knowledge exchange, and requires the effective operation of two-way channels of communication. It is by creating a shared, safe and trusted discussion space within which members can freely operate that a coordination team can assist knowledge exchange. This provides opportunities to answer the questions being asked by the policy and practice communities. Initially, some researchers may not appreciate the need for this; if so, a coordination team can provide the training to enable them to understand the need. For knowledge exchange, such translation is often a highly significant (but often underappreciated) skill that balances what researchers want to say about what they do and what, for instance, policymakers and other decision makers are prepared to read.

\section{Lesson 7: Sharing skills and perspectives}

As Box 1 indicates, a coordination team needs a mix of different skills and different perspectives. This is one reason why the ARCC coordination team was held to be effective, because it had team members with widely diverse backgrounds and varying experience that brought different perspectives. This experience included UK and Canadian government policymaking, social housing regeneration, climate change research, communications, adaptation in the transport sector, Australian government communications, building services, and visual design/website management. Between them, this range of experience allowed team members to bring a breadth of 
understanding of the various political, cultural and social landscapes that apply in the different domains within and across which they were being asked to operate.

\section{Lesson 8: Curating knowledge}

To operate effectively, a coordination team needs to develop a highly bespoke academic-practice interface, and it also has to get researchers to the point where they can present themselves meaningfully to industry members. This may be something that some of them are not used to doing. Equally, team members have to act in reverse - providing opportunities for other stakeholders to raise and sustain their dialogues in a meaningful manner with members of the research community. Team members can also put researchers and non-academics under the right spotlight by curating the right event at the right time. A number of skills have to be exercised here, such as bringing together the right knowledge, creating the right opportunity with a conducive atmosphere, and putting both industry professionals and academics at ease in order to enable them to focus their energies on interacting meaningfully with each other.

\section{Lesson 9: Exploiting network expertise}

Coordination teams need to exploit their own and their network members' expertise to meet externally set initiatives and imperatives. In the UK, the Finch Report (Finch, 2012) set out the principle that the findings of all publicly funded research should be freely accessible in the public domain. The EPSRC set a deadline of May 2015 for achieving this, which was (over) ambitious since, as the ARCC coordination team noticed, this was not an issue that most academic researchers (or their collaborative partners) were working on at all. The coordination team lifted the lid on this box, and put the issue directly in front of its stakeholders by creating guidance for authors on its website in a section called 'OpenARCC' (ARCC, n.d.). As this example illustrates, coordination teams need to scan the horizon continually for their own appropriate initiatives and imperatives, and mobilize the expertise available to meet them, as and when necessary.

\section{Lesson 10: Learning through monitoring and evaluation}

One aspect that has become increasing important for coordination teams is how to assess and capture the impact of a network itself, and how this can inform future work. This goes beyond evaluating the number of people at an event, or tracking website hits, or disentangling the achievements of individual research projects and stakeholders. The ARCC coordination team invested time and effort in developing its own evaluation framework, focused not just on inputs and outputs from the network, but on the outcomes and (where possible) the impacts that could be attributed to the network as a result of the knowledge-exchange activities it supported. Their experience suggests that it is definitely worth allocating resources to go beyond collecting the usual (hard) quantitative metrics, and to spend time on collating softer information in order to be able to track how the results of the networks' activities have added value to the researchers' results. Coordination teams, and those who fund knowledgeexchange activities, need to recognize that this does take time, effort and resources. However, possession of such an evaluation framework can help a team to assess the performance of its network by capturing both qualitative and quantitative information on activities, employing process and output indicators and metrics, followed up by outcome and impact indicators. 


\section{Conclusion}

A driving premise underpinning the ARCC network was that knowledge exchange between academic researchers, policy and practice communities is crucial to delivering expected benefits across the UK economy and society by improving the timing and quality of engagement across the academic-industry divide. The exchange of knowledge between research, policy and practice communities can be mutually supportive, and when done through an effective knowledge-exchange network can achieve results that no one individual or group could achieve working alone. In pushing this agenda, the coordination team for the ARCC network championed the need for flexibility in how it operated (changing the scope and focus of its activities to reflect the changing needs of both academic partners and their collaborating partners). Part of this involved identifying, and then taking advantage of, opportunities as they arose, responding to the needs of stakeholders, and working at the boundary of research, policy and practice.

The lessons about knowledge exchange that are offered in this paper are selfevidently important for knowledge-exchange professionals. But they are also vital to research funders, policymakers and the end users of research findings, if they wish to improve the social and economic benefits of their investments (whether in terms of time or value for money). And they are also important to individual researchers, who need to understand how, when and where the results of their research can have greater real-world influence.

Building capable and compelling knowledge-exchange coordination needs sustained investment requiring adequate and dedicated resources. The array of technical and interpersonal skills required for such coordination is unlikely to reside in a single, or even in a small number of, individuals. Knowledge-exchange coordination teams need to be structured to deliver the diverse set of skills required to effectively support the two-way knowledge-exchange process that engages research, policy and practice communities. Building such teams requires commitment to the longterm development of knowledge-exchange skills. And this, in turn, requires funding arrangements that do not stop at the shorter-term funding given, not just to individual research projects, but even to longer-term research programmes and portfolios. Knowledge-exchange funding in the UK, as elsewhere, needs to recognize the longitudinal nature of knowledge exchange as an activity, and the continuous call on resources that this requires. It would also benefit from recognizing the mix of technical and social skills and capabilities that effective knowledge exchange needs to call upon. And this, in turn, should be reflected in the development of both quantitative and qualitative (and sometimes intangible) metrics for assessing knowledge-exchange performance.

In the development of knowledge-exchange programmes there needs to be an explicit recognition of the range of activities involved (as exemplified by the ARCC network). These include: creating a safe, shared space to enable opportunities for engagement and the building of trust between researchers, policy makers and practitioners; translating and communicating science for practical decision making; understanding of the varied and changing needs of researchers and stakeholders; and knowing how to manage these ingredients into a successful mix. A knowledgeexchange network that operates in this way - especially if it can manage to be more than just the sum of these parts - should be able to increase the quality and relevance of the research investments that it supports, and increase the impact of those investments for the benefit of the economy and society for the country/countries that fund it. 


\section{Acknowledgements}

This paper arises out of research funded by the UK Engineering and Physical Sciences Research Council (Grant Number EPSRC: EP/L005700/1). The authors would like to thank all the network members and coordination team members who shared their experiences in contributing to this work:

- Arup

- Chartered Institution of Building Services Engineers

- Department for Environment, Food and Rural Affairs

- Engineering and Physical Sciences Research Council (EPSRC)

- Infrastructure Operators Adaptation Forum

- University College London

- University of Oxford.

Particular thanks to Roger Street and Philip Sivell for their considered comments, and to Stephanie Ferguson for her supreme editing skills.

\section{Notes on the contributors}

Tanya Wilkins was Communications Manager for the UK Climate Impacts Programme at the University of Oxford. She is a promoter of genuine communication and engagement, and an advocate for the experts in these new days of 'Google-fuelled online warriors'. She is currently in Australia working on a PhD in science communication (@wiltm001).

Ian Cooper is an independent research consultant at Eclipse Research. His expertise focuses on the design, management, monitoring and impact assessment of research programmes, portfolio and projects, especially those relating to the built environment and sustainable development.

\section{References}

ARCC (Adaptation and Resilience in the Context of Change) (n.d.) 'OpenARCC'. Online. https://tinyurl.com/yxlormdu (accessed 31 May 2019).

BIS (Department for Business, Innovation and Skills) (2016) 'John Kingman to lead creation of new f6 billion research and innovation body'. Press release, 17 May. Online. https://tinyurl.com/ z9oo9tz (accessed 31 May 2019).

Breckon, J. and Dodson, J. (2016) Using Evidence: What works? London: Alliance for Useful Evidence. Online. https://tinyurl.com/y5kzh98s (accessed 2 June 2019).

Cooper, I. (1993) Good Practice in Information Dissemination. Rev. ed. Swindon: Science and Engineering Research Council. Online. https://tinyurl.com/y3b9zm64 (accessed 31 May 2019).

Dilling, L. and Lemos, M.C. (2011) 'Creating usable science: Opportunities and constraints for climate knowledge use and their implications for science policy'. Global Environmental Change, 21 (2), 680-9.

EPSRC (Engineering and Physical Sciences Research Council) (2014) EPSRC Review of the SUE and ARCC Programmes. Swindon: Engineering and Physical Sciences Research Council. Online. https://tinyurl.com/yyv7fue7 (accessed 31 May 2019).

Fazey, I., Evely, A.C., Reed, M.S., Stringer, L.C., Kruijsen, J., White, P.C.L. et al. (2013) 'Knowledge exchange: A review and research agenda for environmental management'. Environmental Conservation, 40 (1), 19-36.

Finch, J. (2012) Accessibility, sustainability, excellence: how to expand access to research publications. Report of the Working Group on Expanding Access to Published Research Findings.

Gagnon, M.L. (2011) 'Moving knowledge to action through dissemination and exchange'. Journal of Clinical Epidemiology, 64 (1), 25-31. 
Glaser, B.G. (ed.) (1993) Examples of Grounded Theory: A reader. Mill Valley, CA: Sociology Press. HEFCE (Higher Education Funding Council for England) (2015) 'Evaluations of HEFCE funding for knowledge exchange: The Higher Education Innovation Fund'. Online. https://tinyurl.com/ y2wh72cy (accessed 31 May 2019).

Johnson, J. (2017) 'In full: Jo Johnson speech to the HEFCE Annual Conference 2017'. Times Higher Education, 12 October. Online. https://tinyurl.com/y4pwlf26 (accessed 31 May 2019).

KTN (Knowledge Transfer Network) (2019) 'The Future. Faster.' Online. https://innovation.ox.ac.uk (accessed 26 June 2019).

Oxford University Innovation (n.d.) 'We help Oxford ideas come to life'. Online. https://innovation. ox.ac.uk (accessed 16 January 2019).

RAE (Research Assessment Exercise) (2008) 'About the RAE 2008'. Online. www.rae.ac.uk/aboutus/ (accessed 16 January 2019).

RCUK (Research Councils UK) (2010) RCUK Review of Pathways to Impact: Summary. Swindon: Research Councils UK. Online. https://tinyurl.com/yxf6k89f (accessed 2 June 2019).

REF (Research Excellence Framework) (2019) 'What is the REF?'. Online. www.ref.ac.uk/about/ whatref/ (accessed 31 May 2019).

Research England (2019) 'Knowledge Exchange Framework (KEF): Consultation'. Online. https://tinyurl.com/yb4agv8h (accessed 31 May 2019).

Revans, R.W. (1982) 'What is action learning?'. Journal of Management Development, 1 (3), 64-75.

Robertson, K. (2005) 'Active listening: More than just paying attention'. Australian Family Physician, 34 (12), 1053-5.

Schön, D.A. (1983) The Reflective Practitioner: How professionals think in action. New York: Basic Books.

Tetroe, J.M., Graham, I.D., Foy, R., Robinson, N., Eccles, M.P., Wensing, M., Durieux, P., Légaré, F., Nielson, C.P., Adily, A., Ward, J.E., Porter, C., Shea, B. and Grimshaw, J.M. (2008) 'Health research funding agencies' support and promotion of knowledge translation: An international study'. Milbank Quarterly, 86 (1), 125-55.

Trehan, K. and Pedler, M. (2011) 'Cultivating foresight and innovation in action learning: Reflecting ourselves; reflecting with others'. Action Learning: Research and Practice, 8 (1), 1-4.

UKCIP (UK Climate Impacts Programme) and Cooper, I. (2016) Lessons from Coordinating a Knowledge Exchange Network: ARCC report. Oxford: UK Climate Impacts Programme.

UKRI (UK Research and Innovation) (n.d.(a)) 'Working with business'. Online. www.ukri.org/ innovation/working-with-business/ (accessed 27 June 2019).

UKRI (UK Research and Innovation) (n.d.(b)) 'Delivering economic impact'. Online. https://tinyurl. com/y6ovem8w (accessed 2 June 2019).

Von Glasersfeld, E. (1989) 'Cognition, construction of knowledge, and teaching'. Synthese, 80 (1), 121-40. 